\title{
Cost Effectiveness of New Diagnostic Tools for Cutaneous Leishmaniasis in Afghanistan
}

\author{
Céline Aerts $^{1} \cdot$ Martijn Vink $^{2} \cdot$ Sayed Jalal Pashtoon ${ }^{3} \cdot$ Sami Nahzat $^{4} \cdot$ Albert Picado $^{5} \cdot$ Israel Cruz $^{5} \cdot$ Elisa Sicuri $^{1,6}$
}

Published online: 21 November 2018

(c) The Author(s) 2018

\begin{abstract}
Background and Objectives Cutaneous leishmaniasis is responsible for chronic and disfiguring skin lesions resulting in morbidity and social stigma. The gold standard to diagnose cutaneous leishmaniasis is microscopy but has a variable sensitivity and requires trained personnel. Using four scenarios, the objective of this study is to compare the cost effectiveness of microscopy with two new tools: Loopamp ${ }^{\mathrm{TM}}$ Leishmania Detection Kit (LAMP) and CL Detect ${ }^{\mathrm{TM}}$ Rapid Test (RDT).

Methods Data related to the cost and accuracy of these tools were collected at the clinic of the National Malaria and Leishmaniasis Control Program in Kabul, Afghanistan. The effectiveness estimates were measured based on the tools' performance but also indirectly, using the disability-adjusted life years. A decision tree was designed in TreeAge Healthcare Pro 2016, combined with a Markov model representing the natural history of cutaneous leishmaniasis. In addition to a deterministic analysis, univariate sensitivity and probabilistic analyses were performed to test the robustness of the results.

Results If the tools are compared at the National Malaria and Leishmaniasis Control Program level in a period of low incidence, microscopy remains the preferred option. LAMP becomes more appropriate during cutaneous leishmaniasis seasons or outbreaks when its capacity to process several tests (e.g. up to 48) at a time can be maximised. RDT has a cost similar to microscopy when used at the reference clinic but as it is relatively easy to use, it could be implemented at the peripheral level, which would become cheaper than employing microscopy at the reference clinic. Moreover, combining RDT with microscopy or LAMP at the reference clinic for the negative suspects is economically more interesting than directly performing LAMP or microscopy respectively on all cutaneous leishmaniasis suspects at the reference clinic.
\end{abstract}

Conclusions When taking advantage of their respective strengths, LAMP and RDT can prove to be cost-effective alternatives to using microscopy alone at the reference clinic.

Electronic supplementary material The online version of this article (https://doi.org/10.1007/s40258-018-0449-8) contains supplementary material, which is available to authorized users.

Céline Aerts

celine.aerts@isglobal.org

1 ISGlobal, Hospital-Clínic-Universitat de Barcelona, Carrer Rosselló 132, 08036 Barcelona, Spain

2 Health Works (Formerly HealthNet TPO), Amsterdam, The Netherlands

3 Health Works (Formerly HealthNet TPO), Kabul, Afghanistan

4 National Malaria and Leishmaniasis Control Program, Ministry of Public Health, Kabul, Afghanistan

5 Foundation for Innovative New Diagnostics, Geneva, Switzerland

6 Health Economics Group, Department of Infectious Disease Epidemiology, School of Public Health, Imperial College London, London, UK

\section{Key Points for Decision Makers}

If the diagnoses are to be made at a reference clinic in a period of low incidence, it is not worth replacing microscopy with the novel tools.

The Loopamp ${ }^{\mathrm{TM}}$ Leishmania Detection Kit (LAMP) is particularly relevant during cutaneous leishmaniasis seasons or outbreaks when its capacity to process several tests at a time is used (i.e. minimum of 35 tests).

The characteristics of the CL Detect ${ }^{\mathrm{TM}}$ Rapid Test (RDT) make its implementation feasible in peripheral health centers. A primary screening with RDT in peripheral centers followed by LAMP or microscopy at the reference clinic for the negative suspects is more cost effective than screening all suspects at the reference clinic directly with LAMP or microscopy respectively. This is conditional on the fact that follow-up treatments are carried out in peripheral centers as well. 


\section{Introduction}

The leishmaniases are a group of infections caused by protozoan parasites of the Leishmania genus that are transmitted to humans through the bites of infected female phlebotomine sandflies. There are three main forms of leishmaniasis: visceral, mucocutaneous and cutaneous. Cutaneous leishmaniasis (CL) is the most common form of the disease and produces lesions on exposed parts of the body [1]. Although not fatal, it is responsible for chronic and disfiguring skin lesions resulting in high morbidity and social stigma [2,3]. More than 100,000 new cases of CL are reported annually to the World Health Organization in the Eastern Mediterranean Region. Nevertheless, the incidence is estimated to be three to five times higher as most cases are either undiagnosed or not reported to health authorities [4].

Afghanistan is one of the countries with the highest prevalence of CL, caused by Leishmania major and Leishmania tropica [5]. The latter is the most prevalent and is related to anthroponotic urban transmission. It can evolve into cutaneous leishmaniasis recidivans (CLR) characterised by papular lesions appearing around the scar of a healed lesion months to years after a clinical cure, which may last for many years $[4,6]$. Although efforts have been made to rebuild the Afghan healthcare system after the fall of the Taliban regime in 2001, the country is struggling with insecurity, corruption, low-quality health services and accessibility to health services [6]. Accordingly, cost-effective solutions to tackle public health priorities are needed, and as for leishmaniasis specifically, significant improvements in the diagnostic and treatment strategies are necessary.

The main diagnostic tools for CL are microscopy and polymerase chain reaction (PCR) but both have their respective drawbacks. Microscopy, considered as the mainstay diagnosis method, requires trained personnel and has a low and variable sensitivity, which, in many cases, leads clinicians to neglect its use and reach a diagnosis based on clinical judgements [7]. However, the broad variety of CL manifestations complicates its clinical diagnosis and its identification among other infectious and non-infectious diseases such as psoriasis, blastomycosis, chromoblastomycosis, sarcoidosis, and cutaneous tuberculosis in the Eastern Mediterranean Region. Additionally, in long-lasting lesions, the lesion may expand but the parasite load decreases over time, which makes its detection more difficult. In such cases, molecular diagnosis (i.e. PCR) has shown to be far more sensitive than microscopy $[8,9]$. Nevertheless, this tool requires wellequipped laboratory facilities and experienced laboratory staff, as well as sufficient financial resources, which prevents its use outside well-equipped laboratories [10].

Thus, there is a need to move towards user-friendly, costeffective and field-amenable diagnostic options. This need is further heightened by the current treatment options for CL. Treatment regimens are not standardised; the first-line treatment in Afghanistan is based on injectable pentavalent antimony, which is usually intra-lesional but may require systemic (intramuscular) administration in complicated cases such as CLR. The daily intramuscular injections impose significant travel costs and commuting time to the patients. Although effective, these injections can be toxic and cause serious side effects [11,12]. Accordingly, accurate diagnosis will ensure that only those infected will be given treatment, avoiding unnecessary and unpleasant treatment, the misuse of available drugs and the emergence of drug resistance.

In a context such as Afghanistan where skilled health workers are lacking, improving technology can help increasing labour productivity and the quality of CL detection. Two point-of-care diagnostic tools have been recently developed: Loopamp ${ }^{\mathrm{TM}}$ Leishmania Detection Kit (LAMP) [Eiken Chemical Co., Japan] and CL Detect ${ }^{\mathrm{TM}}$ Rapid Test (RDT) [InBios International Inc., USA] (a detailed description of these tools is given in the Electronic Supplementary Material $[\mathrm{ESM}])$. On the one hand, LAMP is able to perform as well as PCR in terms of sensitivity and specificity but the reagents come in a ready-to-use dry format that is stable at ambient temperature. The results are obtained faster and can be visualised directly using simple detection methods [13]. Additionally, LAMP can process several tests at a time, from 8 to 48 or more, depending on the machine. On the other hand, RDT is fast and easy to use, does not require any machine as opposed to LAMP and microscopy, and has a close to perfect specificity [14].

To our knowledge, there is no cost-effectiveness study comparing the available diagnostic tools for CL; the available cost-effectiveness studies on CL tend to focus on treatment strategies instead [15-19]. To fill this gap, the objective of this study is to compare the cost effectiveness of RDT and LAMP with that of microscopy, using PCR as a reference [20-22]. We use four hypothetical scenarios. Scenario 1 compares the above tools in a reference clinic, the National Malaria and Leishmaniasis Control Program (NMLCP), assuming one test is performed at a time, whereas scenario 2 compares the same tools in the same clinic but assumes a high incidence of CL (e.g. winter season) where the assumed full capacity of the LAMP is being used (48 tests processed at a time). Scenario 3 attempts to capture the benefit of implementing RDT in remote healthcare facilities compared to the implementation of LAMP and microscopy in the reference clinic. In this scenario, treatment is administered in remote facilities, thus diminishing the associated treatment costs to the patient by reducing commuting times and expenses. Last, scenario 4 relies on the same assumptions, except that negative RDT suspects are tested again at the NMLCP with microscopy or LAMP. 


\section{Data and Methods}

\subsection{Study Population and Diagnosis}

The new diagnostic tools were evaluated among 274 individuals presenting themselves with suggestive signs of CL at the leishmaniasis clinic of the NMLCP in Kabul; a department of the Ministry of Public Health. The clinic is the CL reference clinic in Kabul treating 5000-7000 new CL cases per year. To be enrolled in the study, inclusion criteria were: (1) older than 2 years of age; (2) consenting to participate; and (3) not receiving treatment for CL at the time of enrolment. Samples from participants were subject to the four diagnostic tests: microscopy, LAMP, RDT and PCR (see details in the ESM). A logistic regression was performed to assess whether individual-level characteristics, age and sex were significantly associated with being positive for CL (when presenting skin lesions). The recruitment period spanned from April to June 2016.

\subsection{Cost-Effectiveness Model Structure}

We developed a decision tree designed in TreeAge Healthcare Pro 2016 (TreeAge Software, Williamstown, MA, USA), which was combined with a Markov model representing the natural history of CL due to L. tropica (see Figs. 1 and 2), as all patients have shown to be infected with this Leishmania species. The model is based on a static cohort of 10,000 individuals and runs for 80 annual cycles. Individuals enter the model at the age of 6 years: the youngest age observed in the study population. To follow the cohort over a life-long span, the individual entering the model encounters an annual probability of dying, which is independent of CL and varies across age groups and sex. The Markov model is composed of seven mutually exclusive health states and starts with "no skin lesion", "skin lesion(s)" and "death" states to translate this health facility-based study into a community-based study and to capture the risk of infection in an endemic area. The remaining health states include the "No CL", "CL", "cure" and the "CLR" states (see Fig. 2).

Each annual cycle, the individual either stays in the same health state or moves to another one according to transition probabilities (see Table 4). If the individual is healthy and does not have skin lesions, he/she can: (1) remain healthy; (2) develop skin lesion(s); or (3) die for reasons independent of CL. If the individual presents with skin lesion(s), the lesions might be due to: (1) CL, which is equal to the percentage of confirmed CL cases observed at the health facility or (2) other diseases (i.e. "No CL"). Alternatively, the individual may die for unrelated reasons. Whether the CLpositive cases are detected will not only depend on the sensitivity of the tools but also on the likelihood that infected people seek a diagnosis. What follows after a true-positive or a false-negative case is best explained in Fig. A1 in the ESM.

If a patient is infected by L. tropica and develops CL, he/ she can either: (1) remain infected for another year; $[4,10]$ (2) become cured; or (3) die. If a patient is not infected with L. tropica (i.e. "No CL"), he/she can either: (1) stay uninfected for another cycle; (2) become infected and develop $\mathrm{CL}$ according to the incidence rate; (3) or die. Once the patient has cured from a CL infection (i.e. "Cure"), he/she can: (1) stay cured; (2) develop CLR ("CLR" state) [23]; or (3) die. Patients infected with CLR can: (1) stay infected with CLR for up to 10 years; [4] (2) become cured; or (3) die. Nonetheless, it is worth highlighting that no cases of CLR were recorded among the study population.

\subsection{Cost Estimates}

Data on costs were collected through three tailor-made questionnaires capturing both the patient and the health system perspective. Costs figures were initially collected in local currency (i.e. Afghan Afghani) and were then translated into US dollars using the exchange rate for the year 2016 (i.e. 0.015 ). First, the 'patient cost questionnaire' (see questionnaire I in the ESM) was administered to a subset $(n=111)$ of the 274 individuals enrolled in the study, regardless of their diagnostic results. The first half of the questionnaire was completed on the day of the diagnosis and the remaining half was completed at the end of the treatment period by positive patients only, with the help of a fieldworker. This questionnaire gathers information related to direct (i.e. transportation) and indirect (i.e. wage loss during travelling and incapacity to work during illness period) costs associated with a potential CL episode. Patient costs were controlled for individual-level characteristics (i.e. age, sex and occupation) by matching cost estimates obtained from this questionnaire to a patient folder that collected information on individual characteristics such as occupation and family income. This led to a reduced subset of 85 individuals. Second, the 'laboratory and medical staff' questionnaire (see questionnaire II in the ESM) captures cost estimates among medical staff running CL diagnostics. These include the time spent on average per diagnostic; the types of medical staff required and their salaries; and the equipment required to run the diagnostic. The market price of the kits and the cost of the machines/instrument (i.e. incubator/thermocycler in the case of LAMP) were also included. The cost of the instruments was calculated over 80 years, the cohort life expectancy, assuming a life span of 5 years. DNA extraction cost was included for LAMP. Last, an additional questionnaire was administered to medical staff: the 'drug and treatment' questionnaire (see questionnaire III in the ESM), which collected information mainly related to costs of intra-lesional and intramuscular treatment. Treatment cost is based on the 


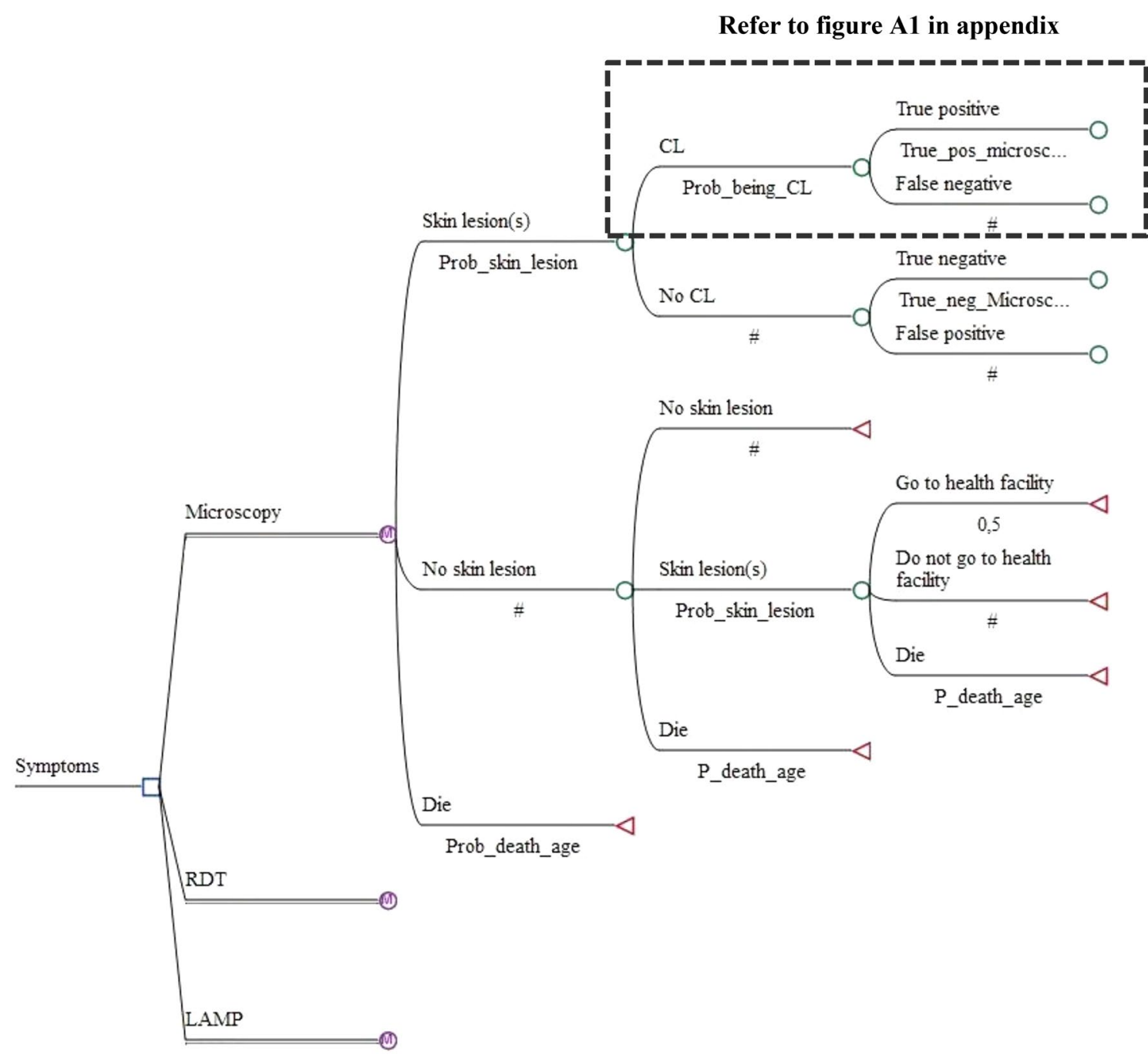

Fig. 1 Decision tree: comparative strategies. $C L$ cutaneous leishmaniasis, LAMP Loopamp ${ }^{\mathrm{TM}}$ Leishmania Detection Kit, RDT CL Detect ${ }^{\mathrm{TM}}$ Rapid Test

generic price of Pentostam ${ }^{\circledR}$, which is donated by the World Health Organization in Afghanistan [24]. Patients pay outof-pocket-associated travel expenses and incur a wage loss from travelling and waiting to receive the daily injections. If treatment was intra-lesional, patients would have to receive 3-5 injections, whereas if treatment was intramuscular, patients would have to receive 14-21 injections implying considerable travel expenses. Lastly, all cost parameters were discounted at $3 \%$ per annum as recommended by World Health Organization guidelines [25].

\subsection{Effectiveness Estimates}

In the economic model, the effectiveness estimates were estimated directly through the sensitivity and specificity of the tools, as presented in Table 1, but also indirectly through an indicator of disease burden: the disability-adjusted life years
(DALYs). The DALYs were estimated per annual cycle and because CL is not lethal these are an estimation of years lived with disability. To better understand and capture the impact of a CL episode on a patient's quality of life, the standardised 'Dermatology Life Quality Index' (DLQI) questionnaire was administered to individuals enrolled in the study and used as an indicator of social stigmatisation [26] (see questionnaire IV in the ESM). Information collected through the DLQI informed the calculation of DALYs: more precisely, the percentage of CL-positive people (based on PCR) who reported "A little", "A lot" or "Very much" embarrassment and/or social stigmatisation in question 2 were attributed a higher disability weight of 0.067 . This disability weight is coded as disfigurement level 2 in the Global Burden of Diseases study and is defined as follows: "has a visible physical deformity that causes others to stare and comment" [27]. The remainder of the cohort was attributed 
Fig. 2 Natural history of Leishmania tropica

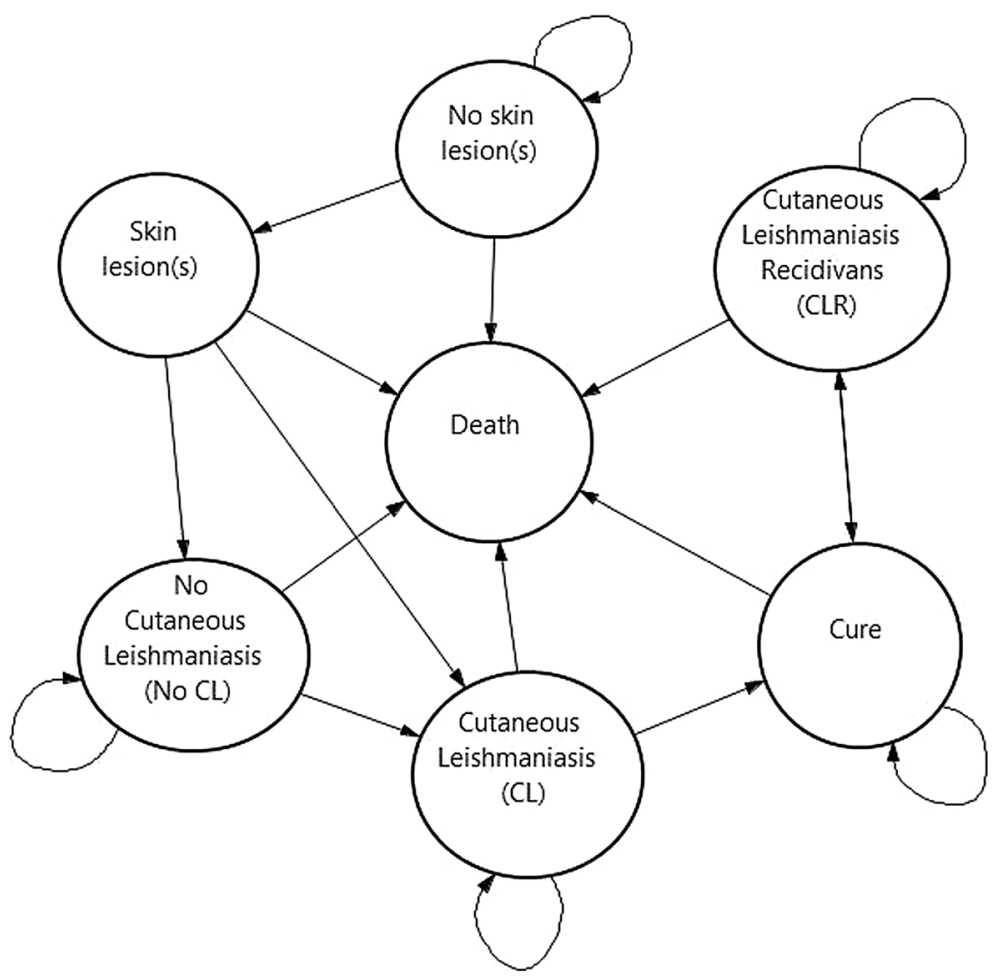

Table 1 Sensitivity and specificity of the tools using polymerase chain reaction as a reference

\begin{tabular}{lll}
\hline Tools & $\begin{array}{l}\text { Diagnostic performance } \\
(\%)\end{array}$ & $95 \% \mathrm{CI}$ \\
\hline Microscopy & $\mathrm{Se}=78.97$ & $73.74-84.20$ \\
& $\mathrm{Sp}=77.27$ & $57.49-97.06$ \\
RDT & $\mathrm{Se}=66.27$ & $60.23-72.31$ \\
& $\mathrm{Sp}=95.45$ & $84.48-100$ \\
LAMP & $\mathrm{Se}=89.68$ & $85.73-93.64$ \\
& $\mathrm{Sp}=63.64$ & $41.26-86.01$ \\
\hline
\end{tabular}

CI confidence interval, LAMP Loopamp ${ }^{\mathrm{TM}}$ Leishmania Detection Kit, $R D T$ CL Detect ${ }^{\mathrm{TM}}$ Rapid Test, $S e$ sensitivity, $S p$ specificity

Source: [29]

a disability weight of 0.011 , which is coded as disfigurement level 1 and defined as "a slight, visible physical deformity that others notice, which causes some worry and discomfort" [27].

As no age weighting and discounting were taken into account in the DALY formulation, DALYs for CL and CLR are simply a weighted average of disfigurement level 1 and 2, which can be accumulated for up to 2 and 10 years, respectively [28]. However, although no discounting was taken into account in the DALY formulation, a discount rate of $3 \%$ per annum was applied in the decision tree as DALYs have a bigger impact in younger ages.

\subsection{Scenarios}

To capture the inherent benefit of the tools, four scenarios were studied. Scenario 1 compared microscopy, LAMP and RDT at the NMCLP level, assuming that the full capacity of the LAMP is irrelevant such that one test at a time is being processed. This is a rather conservative approach but feasible outside the CL incidence peak (i.e. outside the winter season). Scenario 2 compared the tools at the NMLCP level but assuming here that the capacity of the LAMP to process several samples at a time is used fully. To do so, the labour cost spent on LAMP is divided by 48, its assumed maximum capacity. The maximum capacity of the LAMP can vary and hence a sensitivity analysis was conducted on this parameter. Scenario 3 compared microscopy and LAMP at the NMLCP level but RDT at the peripheral level, that is, in remote health facilities. To capture the benefit of implementing the RDT in peripheral facilities, the associated treatment costs to the patient were diminished by half, which are independent of the treatment cost per se, as treatment is provided by the NMLCP, but instead include transportation costs and wage loss as a result of commuting and waiting to receive the daily injections. As halving the treatment-associated costs when RDT is implemented at peripheral levels is rather arbitrary, we conducted a sensitivity analysis on this parameter to look for any potential threshold value(s) that would alter the order of the strategies. Finally, as the sensitivity of RDT is relatively low (i.e. 
a high proportion of false negatives), a fourth scenario was studied in which RDT is implemented at the peripheral level but negative patients are sent to the NMLCP to be tested again with microscopy or LAMP. In scenario 4 (and as in scenario 3 ), we assumed that treatment would be administered at the peripheral level.

\subsection{Data Analysis}

\subsubsection{Measurement of Cost Effectiveness}

An intervention is judged cost effective if the incremental cost-effectiveness ratio (ICER) between two competing strategies is below the country's gross domestic product per capita (US\$561) [30]. If the incremental effectiveness between strategies is close to 0 , the net monetary benefit (NMB) can also be used for comparing strategies, $\mathrm{NMB}=$ threshold $*$ effectiveness - cost, where the weight is put on costs. The strategy with the highest NMB is the one preferred-that is, often the one reporting the lowest cost.

\subsubsection{Model Estimate and Sensitivity Analysis}

The model was estimated by applying deterministic and probabilistic analyses. A deterministic analysis was performed using the mean or median value for each parameter, depending on distribution skewness. Univariate sensitivity was applied to scenario 1 by varying the mean or the median values of all parameters by both $-50 \%$ and $+50 \%$ or to the minimum or maximum feasible values (i.e. 0 or 1 if parameters are probabilities). For the same scenario, threshold analyses were carried out on selected parameters for which a change (i.e. up to $\pm 50 \%$ ) affects the chosen strategy in terms of their respective costs. Probabilistic analysis was performed through Monte Carlo simulations; the number of iterations needed to produce stable results was based on the graphical representation of the average of the cumulative NMB. Different probabilistic distributions were assigned to parameters following indications from the literature and are listed in Table 4 [31]. To account for uncertainty among individual-level cost data, we regressed the logarithm of the cost of being diagnosed on individual-level characteristics: age, sex and occupation. Occupation was divided into four categories: (1) no earnings: "students" and "jobless"; (2) unsecured jobs: "farmers" and "housekeepers"; (3) secured jobs: "army officer", "government official"; and last (4) unknown occupations. Cholesky decomposition among the parameters was performed so that the variance of each parameters and the variability within the parameters (covariance) is kept constant through a multi-normal distribution.

For the sensitivity and specificity of the tools, the difference between the upper and lower limit from the $95 \%$ confidence interval, based on a t Student distribution, was used to calculate the standard deviations. If the difference between the upper and lower limit was too wide to yield positive alpha and beta values, it was reduced until positive parameters were reached. For variables obtained from the literature or at the health facility level, a standard deviation of $20 \%$ was chosen. [32] Results of the probabilistic analyses were graphically presented through the cost-effectiveness plane and acceptability curves. The cost-effectiveness plane plots all Monte Carlo simulations for the two best strategies, with respect to the incremental cost and effectiveness. Acceptability curves are generated using the NMB and show

Table 2 Deterministic results

\begin{tabular}{|c|c|c|c|c|c|c|}
\hline \multirow[t]{2}{*}{ Scenarios } & \multirow[t]{2}{*}{ Strategies } & \multicolumn{2}{|l|}{ Average per person } & \multirow{2}{*}{$\begin{array}{l}\text { ICER } \\
\text { US\$ per DALY } \\
\text { averted }\end{array}$} & \multirow[t]{2}{*}{ Outcome } & \multirow[t]{2}{*}{ NMB } \\
\hline & & Cost (2016 US\$) & DALYs & & & \\
\hline \multirow[t]{3}{*}{1} & Microscopy & 53.79 & 0.0486 & - & Undominated & -81.03 \\
\hline & RDT & 53.91 & 0.049 & - & Dominated & -81.38 \\
\hline & LAMP & 60.18 & 0.0482 & 18614.89 & Undominated & -87.23 \\
\hline \multirow[t]{3}{*}{2} & Microscopy & c.f. 1 & c.f. 1 & - & Dominated & c.f. 1 \\
\hline & RDT & c.f. 1 & c.f. 1 & - & c.f. 1 & c.f. 1 \\
\hline & LAMP (full capacity) & 53.73 & c.f. 1 & - & Undominated & -80.79 \\
\hline \multirow[t]{3}{*}{3} & Microscopy & c.f. 1 & c.f. 1 & 18325.54 & c.f. 1 & c.f. 1 \\
\hline & RDT (peripheral) & 46.32 & c.f. 1 & - & Undominated & -73.79 \\
\hline & LAMP & c.f. 1 & c.f. 1 & 18457.92 & c.f. 1 & c.f. 1 \\
\hline \multirow[t]{4}{*}{4} & Microscopy & c.f. 1 & c.f. 1 & 586.24 & c.f. 1 & c.f. 1 \\
\hline & RDT (peripheral) + microscopy & 52.27 & 0.0511 & - & Undominated & -80.96 \\
\hline & LAMP & c.f. 1 & c.f. 1 & 2699.6 & c.f. 1 & c.f. 1 \\
\hline & RDT (peripheral) + LAMP & 54.59 & 0.051 & - & Dominated & -83.2 \\
\hline
\end{tabular}

c.f. 1 refer to scenario 1, DALYs disability-adjusted life years, ICER incremental cost-effectiveness ratio, LAMP Loopamp ${ }^{\mathrm{TM}}$ Leishmania Detection Kit, $N M B$ net monetary benefit, RDT CL Detect ${ }^{\mathrm{TM}}$ Rapid Test 
Fig. 3 Cost-effectiveness graph with willingness-to-pay (WTP) line. DALYs disability-adjusted life-years, $L A M P$ Loopamp ${ }^{\mathrm{TM}}$ Leishmania Detection Kit, RDT CL Detect ${ }^{\mathrm{TM}}$ Rapid Test

\section{Scenario 1}

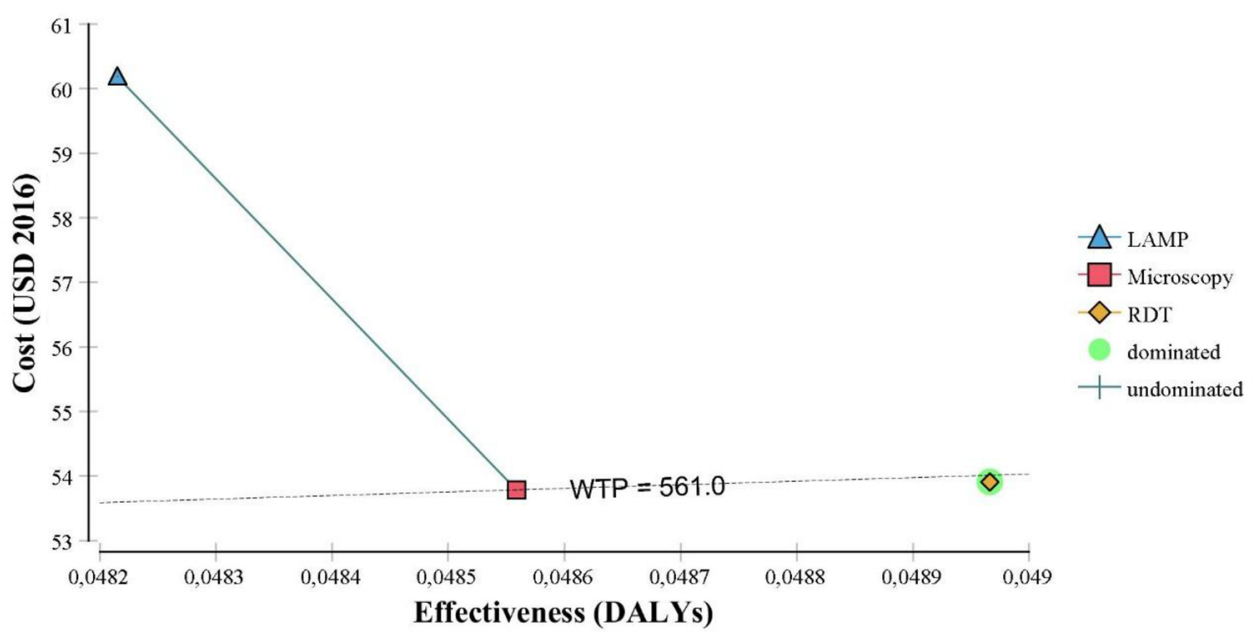

Scenario 2

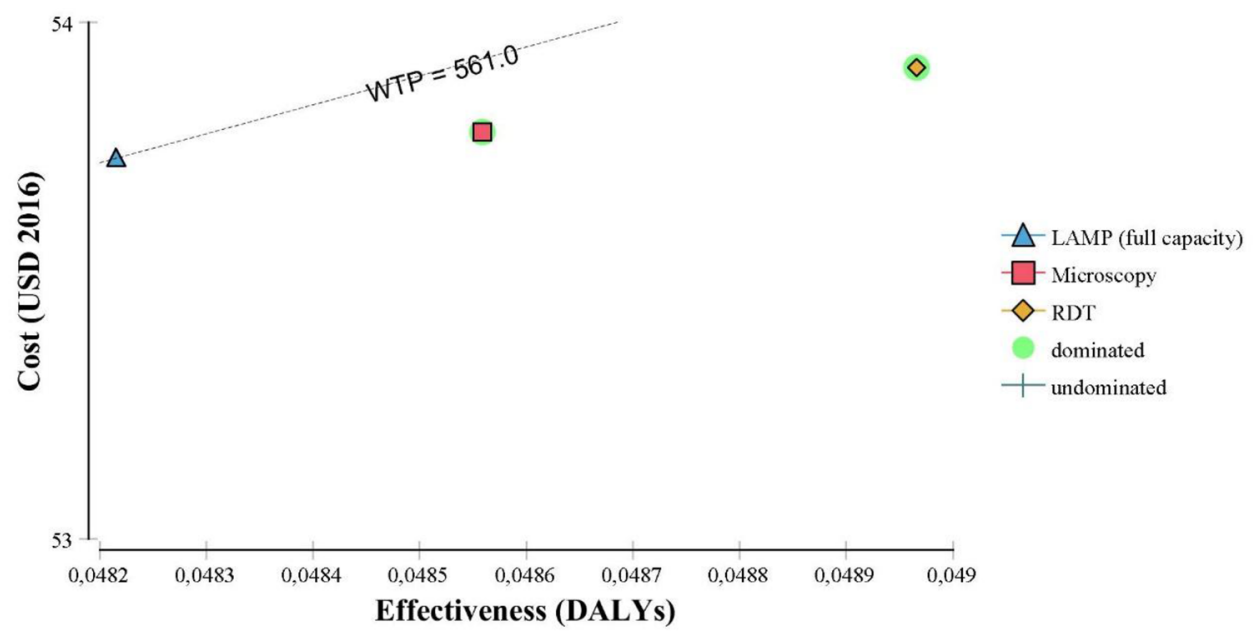

Scenario 3

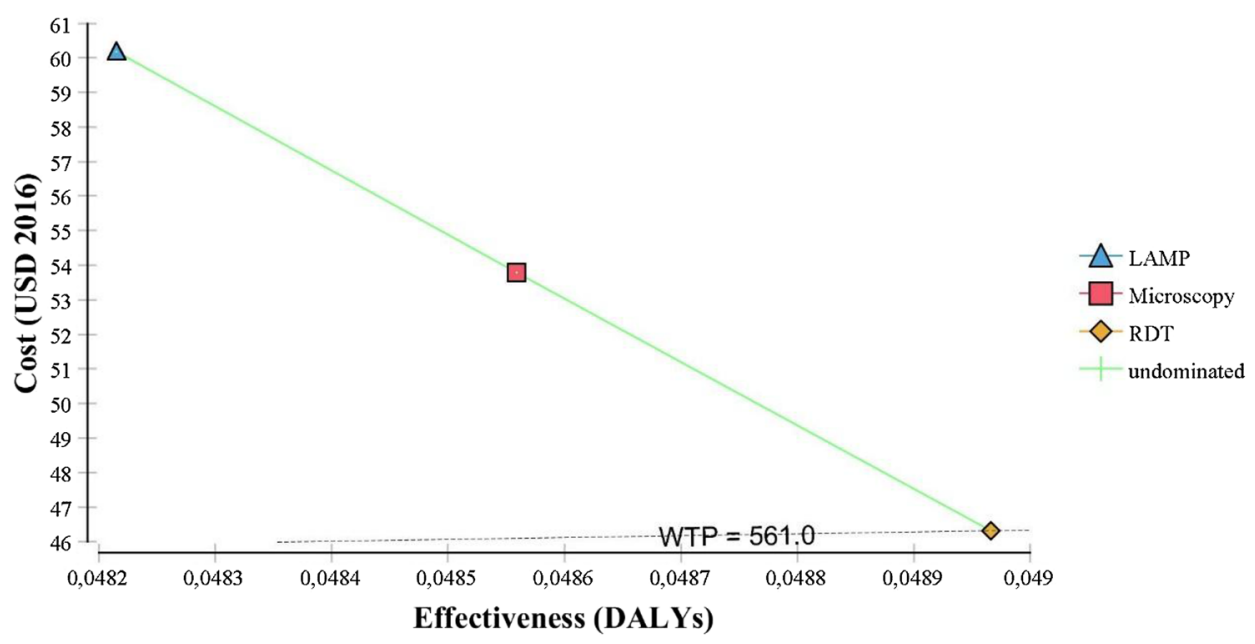


Fig. 3 (continued)

\section{Scenario 4}

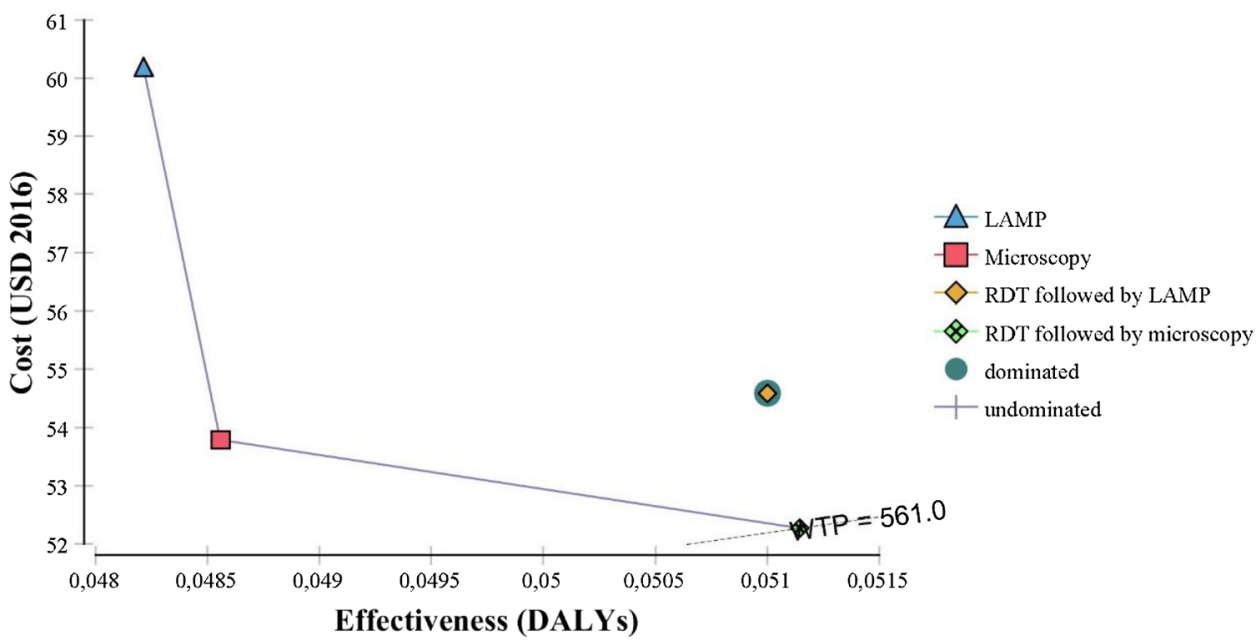

the probability of the tools to be cost effective according to different willingness-to-pay (WTP) values.

\section{Results}

From the logistic regression, it became clear that CL positivity was not significantly correlated with age and sex (refer to Table A1 in the ESM). Hence, the analyses were not stratified across these two demographic variables.

\subsection{Cost}

The predicted mean cost incurred by patients to be tested reaches US\$1 - controlling for individual-level characteristics - and include transportation costs and indirect costs related to wage lost during the clinic visits and the illness period. The results of the regression analyses as well as tests of linear assumptions are presented in Table A2 and Fig. A2 of the ESM. While age and sex are not significantly associated with patients' costs, occupation types are: people with low social security jobs (e.g. house keepers and farmers) experience a $99 \%$ cost increase per CL episode when compared with the reference group: people without any earnings (students and jobless). In contrast, occupations with more social security (e.g. retail workers, tailors) experience a cost increase of 53\% per episode compared with people without earnings. Cost of treatment, borne by the health system, is higher for intramuscular treatment than for intra-lesional treatment, US\$41 vs. US $\$ 13$, but $80 \%$ of the patients are treated with the latter. Travel expenses for the patients are also higher for intramuscular treatment than for intra-lesional treatment, US\$18 vs. US\$4. Microscopy and RDT have identical labour costs, which approximates to US\$14 per person tested. LAMP is slightly more costly with approximately \$US20 per person tested; however, this is using a conservative approach where one test is processed at a time.

\subsection{Disability-Adjusted Life-Years}

Based on the DLQI questionnaire, $60 \%$ of the cohort reported embarrassment and/or social stigmatisation as a result of CL and were attributed a higher disability weight of 0.067 . The remaining $40 \%$ was attributed a lower disability weight of 0.011 . Hence, on average, one episode of CL or CLR was associated with 0.0446 DALY annually, which could be accumulated for up to 2 years for patients infected with CL and 10 years for patients infected with CLR.

\subsection{Cost Effectiveness}

Results of the deterministic analyses are represented in Table 2 for each scenario but also graphically in Fig. 3.

Microscopy and LAMP are the two undominated strategies in scenario 1: the first has the lowest associated cost while the latter has the highest associated effectiveness (i.e. fewer associated DALYs) but as the incremental effectiveness between the two is close to 0 , the ICER tends to infinity. Looking at the NMB, it is almost identical for microscopy 


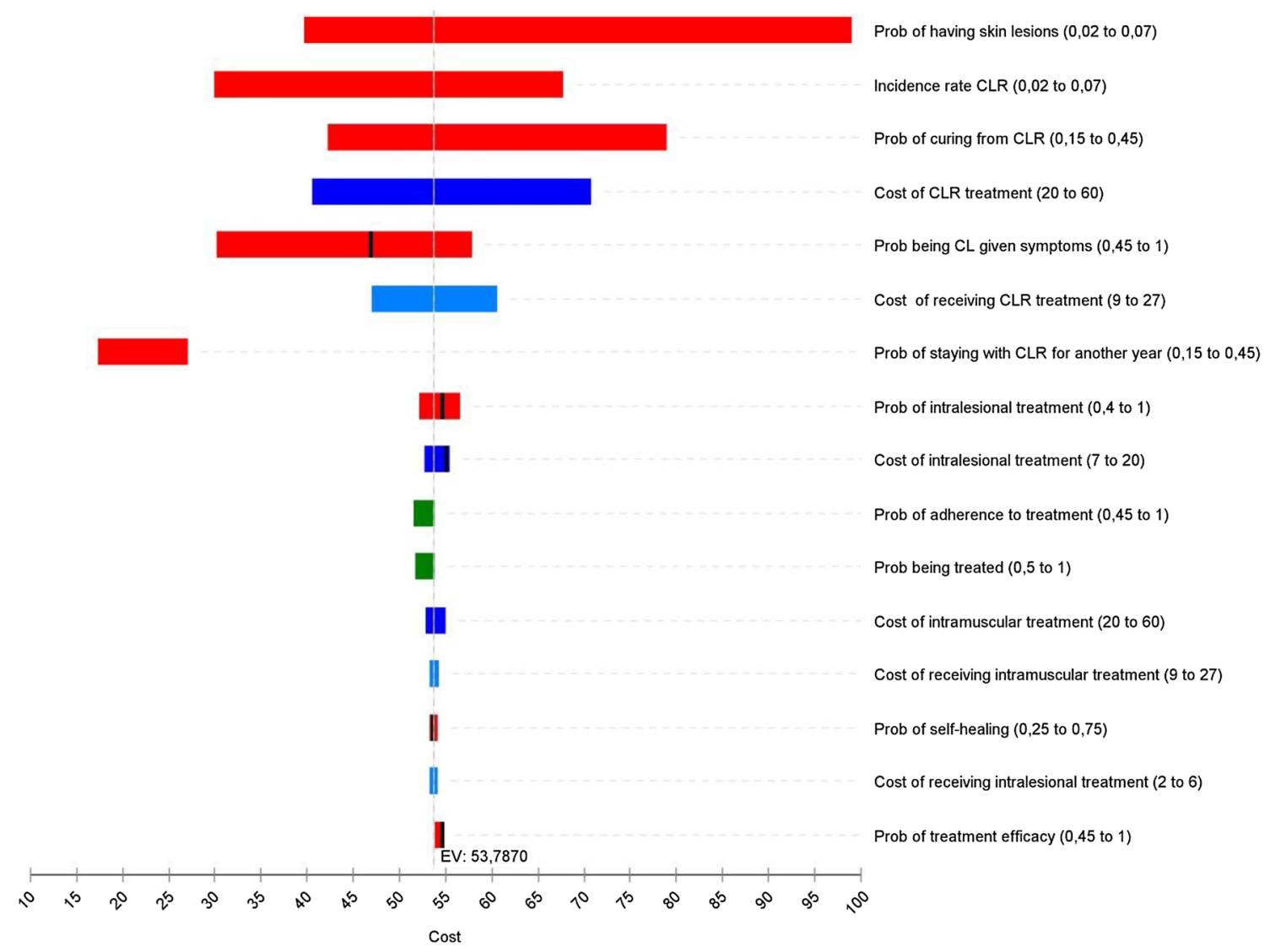

Fig. 4 Tornado diagram. Parameter categories are grouped by color: dark blue represents costs borne by the health system; light blue represents costs borne by the suspects/patients with cutaneous leishmaniasis (CL); red represents transition rates; and green represents patient/clinician behaviours. The values in the parentheses stand for

and RDT but lower for LAMP. In scenario 2, when the assumed full capacity of the LAMP (48 tests at once) is used, LAMP has the lowest cost and the highest effectiveness: it dominates microscopy and RDT with the highest NMB. The sensitivity analysis on the capacity of the LAMP to process several tests at once has shown that: for LAMP to be the cheapest option, 35 tests should be processed at once and for LAMP to have the greater NMB, 17 tests should be processed at once. In scenario 3, RDT has the lowest associated cost and the highest NMB. In other words, if implemented in peripheral health centers, RDT is preferred over microscopy and LAMP used in the reference center. The assumption behind scenario 3 is that if RDT is implemented in remote facilities, the ensuing treatment regimens will be administered in the remote facilities, thereby decreasing the treatment-related costs to the patient. Sensitivity analyses the lower and higher range over which the parameter was varied. The vertical line represents the expected value of the microscopy cost. A segmented bar indicates a change in the cost threshold: CL Detect ${ }^{\mathrm{TM}}$ Rapid Test becomes cheaper than microscopy. CLR cutaneous leishmaniasis recidivans, EV, Prob probability

of the cost of treatment, both intra-lesional and intramuscular, are presented in Fig. A3 of the ESM and shows that a decrease of at least $5 \%$ in the intramuscular treatment cost for patients when RDT is chosen over microscopy or LAMP. When it comes to scenario 4, sending the RDT negatives to the reference clinic to be tested with microscopy or LAMP is naturally more costly than using RDT alone but cheaper than using direct microscopy or LAMP at the NMLCP on all CL suspects. RDT followed by microscopy is cheaper and leads to higher NMB than RDT followed by LAMP.

Figure 3 provides a visual representation of the results where undominated strategies are connected by a line (which does not show in scenario 2 as LAMP dominates both RDT and microscopy). The gradient of this line is the ICER: the steeper the line, the bigger the ICER. A strategy should be chosen if positioned on the ICER line and crossing the WTP 
Table 3 Monte Carlo simulation results

\begin{tabular}{|c|c|c|c|c|c|c|c|}
\hline \multirow[t]{3}{*}{ Scenarios } & \multirow[t]{3}{*}{ Strategies } & \multicolumn{6}{|l|}{ Average per person } \\
\hline & & \multirow{2}{*}{$\begin{array}{l}\text { Cost (2016 \$US) } \\
\text { Mean } \\
{[95 \% \mathrm{CI}]}\end{array}$} & \multirow{2}{*}{$\begin{array}{l}\text { DALYs } \\
\text { Mean } \\
{[95 \% \mathrm{CI}]}\end{array}$} & \multicolumn{2}{|l|}{ Differences } & \multirow{2}{*}{$\begin{array}{l}\text { ICER } \\
\text { US\$ per } \\
\text { DALY } \\
\text { averted }\end{array}$} & \multirow[t]{2}{*}{ Outcome } \\
\hline & & & & Cost (2016 US\$) & DALYs & & \\
\hline \multirow[t]{3}{*}{1} & Microscopy & $56.67[54.48-58.86]$ & $0.0513[0.0493-0.0533]$ & - & - & - & Undom. \\
\hline & RDT & 56.75 [54.55-58.94] & $0.0517[0.0497-0.0537]$ & 0.081 & 0.0004 & - & Dom. \\
\hline & LAMP & $62.86[60.53-65.18]$ & $0.051[0.049-0.053]$ & 6.19 & -0.0003 & $\infty$ & Undom. \\
\hline \multirow[t]{3}{*}{2} & Microscopy & c.f. 1 & c.f. 1 & 0.1286 & 0.0003 & - & Dom. \\
\hline & RDT & c.f. 1 & c.f. 1 & 0.21 & 0.0007 & - & Dom. \\
\hline & LAMP (full capacity) & 56.54 [54.35-58.73] & $0.051[0.049-0.053]$ & - & - & - & Undom. \\
\hline \multirow[t]{3}{*}{3} & Microscopy & c.f. 1 & c.f. 1 & 8.143 & -0.0004 & 6924.40 & Undom. \\
\hline & RDT (peripheral) & 48.53 [46.67-50.38] & $0.0517[0.0497-0.0537]$ & - & - & - & Undom. \\
\hline & LAMP & c.f. 1 & c.f. 1 & 14.33 & -0.0007 & 19797.76 & Undom. \\
\hline \multirow[t]{4}{*}{4} & Microscopy & c.f. 1 & c.f. 1 & 2.20 & -0.003 & $\infty$ & Undom. \\
\hline & $\begin{array}{l}\text { RDT (peripheral) + micros- } \\
\text { copy }\end{array}$ & $54.46[52.51-56.42]$ & $0.0539[0.0519-0.0559]$ & - & - & - & Undom. \\
\hline & RDT (peripheral) + LAMP & $56.74[54.73-58.76]$ & $0.0538[0.0518-0.0558]$ & 2.29 & -0.0001 & - & Dom. \\
\hline & LAMP & c.f. 1 & c.f. 1 & 8.389 & -0.0029 & $\infty$ & Undom. \\
\hline
\end{tabular}

c.f. 1 refer to scenario 1, CI confidence interval, DALYs disability-adjusted life-years, Dom. dominated, LAMP Loopamp ${ }^{\mathrm{TM}}$ Leishmania Detection Kit, RDT CL Detect ${ }^{\mathrm{TM}}$ Rapid Test, Undom. undominated

slope. Accordingly, microscopy should be chosen in scenario 1, LAMP in scenario 2, RDT in scenario 3 and RDT followed by microscopy in scenario 4 .

\subsection{Sensitivity Analysis}

The one-way sensitivity analysis on the expected value of the cost of microscopy vs. RDT and LAMP (with the assumption of scenario 1) is illustrated by the tornado diagram in Fig. 4. To start with transition rate parameters, an increase in the prevalence of skin lesions within the population significantly increases the expected cost of the tools. If the probability of the skin lesions being due to CL ("Prob being CL given symptoms") decreases below 78\%, the expected cost of RDT becomes a slightly lower than microscopy (see Fig. A4 in the ESM). The same applies to the incidence rate of CLR. On the contrary, the greater the annual curing rate the lower the expected cost of the tools and vice versa (i.e. probability of staying with CLR for another year). When lowering the probability of intra-lesional treatment from $80 \%$ to below $70 \%$, RDT becomes cheaper than microscopy (see Fig. A4 in the ESM). The same applies to the probability of treatment efficacy or self-healing: if the former is lower than $45 \%$ or the latter higher than $62 \%$, RDT becomes the cheapest option (see Figs A6 and A7 in the ESM). As with cost parameters, it is obvious that any increase in one of these will result in higher expected costs of the tools. Only one cost parameter can affect the order of the tools when judging on their respective expected costs: the cost of intra-lesional treatment. If increasing above \$US18 per treatment regimen, RDT becomes less costly than microscopy. Finally, as the probability of being treated and the adherence to treatment increase, the cost of the tools increases.

In the probabilistic analysis, about 1000 random iterations of the cost-effectiveness model were required to achieve stable results. Compared with the deterministic results, both the mean cost and mean effectiveness parameters have increased. The mean ICER in most of the scenarios is now tending towards infinity because for many simulations its denominator, the difference in DALYs, is close to zero (see Table 3).

Monte Carlo simulations are represented through the cost-effective plane in Fig. 5 and the cost-effectiveness acceptability curves (CEACs) in Fig. 6, in which the former is generated using the incremental cost and effectiveness and the latter using the NMB. The cost-effectiveness plane compares both the incremental cost and effectiveness of the undominated, or the two best strategies for each scenario. In scenario 1, almost all of the simulations are located above the WTP threshold, suggesting than LAMP is not preferred 
Fig. 5 Cost-effectiveness plane. The circle represents the 95\% ellipse (the $95 \%$ credible interval); the willingness-to-pay (WTP) line represents the WTP threshold that is equal to the one time gross domestic product per capita. DALYs disabilityadjusted life-years, LAMP Loopamp ${ }^{\mathrm{TM}}$ Leishmania Detection Kit, RDT CL Detect ${ }^{\mathrm{TM}}$ Rapid Test
Scenario 1 (LAMP v. Microscopy)

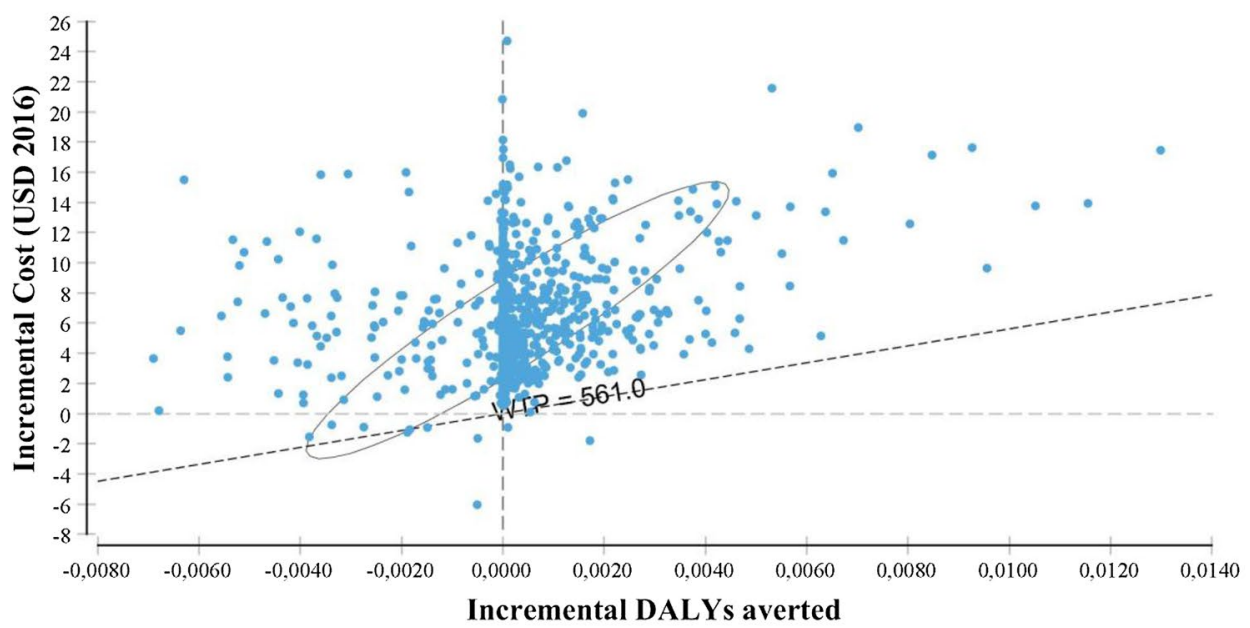

Scenario 2 (LAMP v. Microscopy)

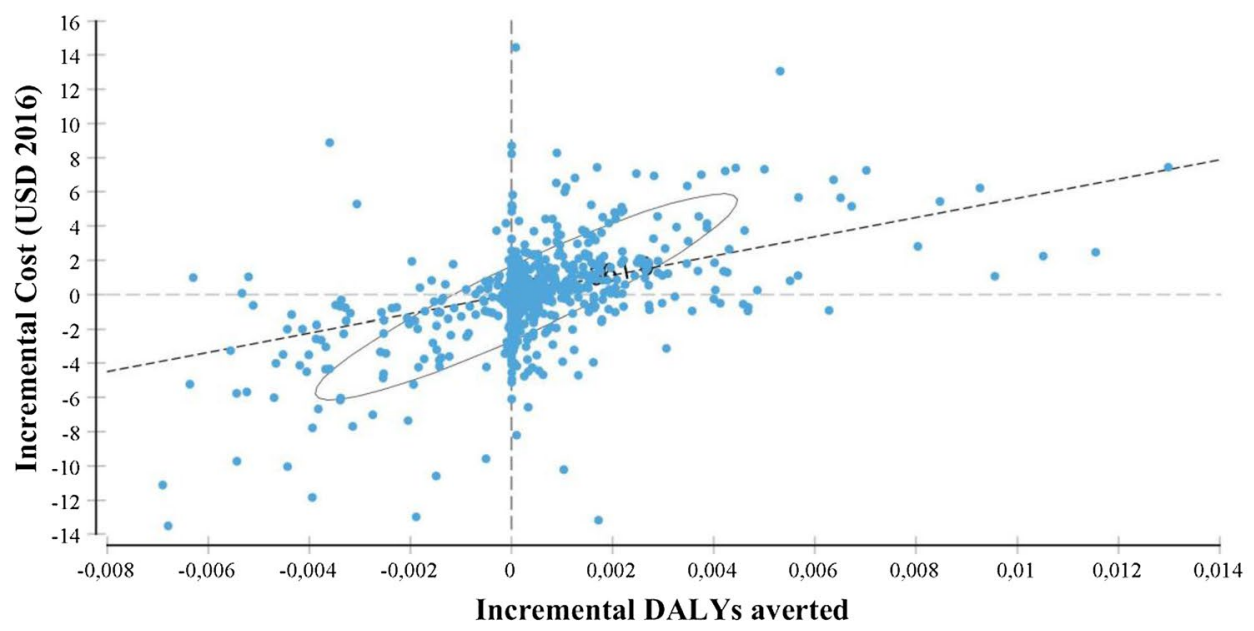

Scenario 3 (LAMP v. RDT)

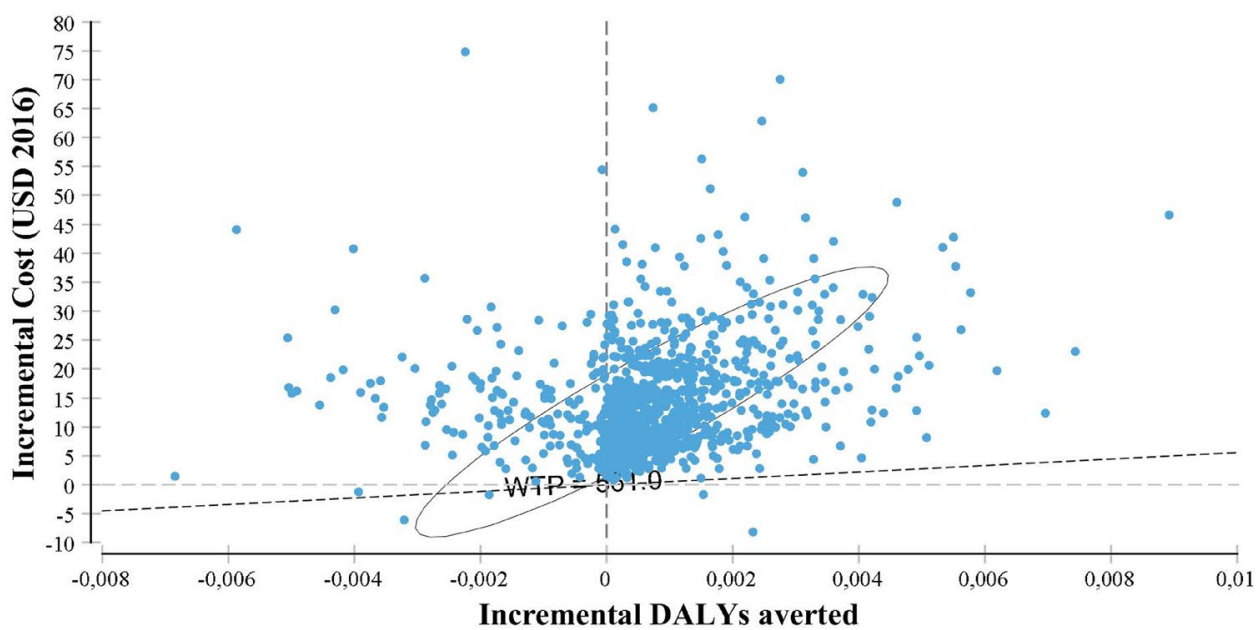


Fig. 5 (continued)

Scenario 4 (LAMP v. RDT followed by microscopy)

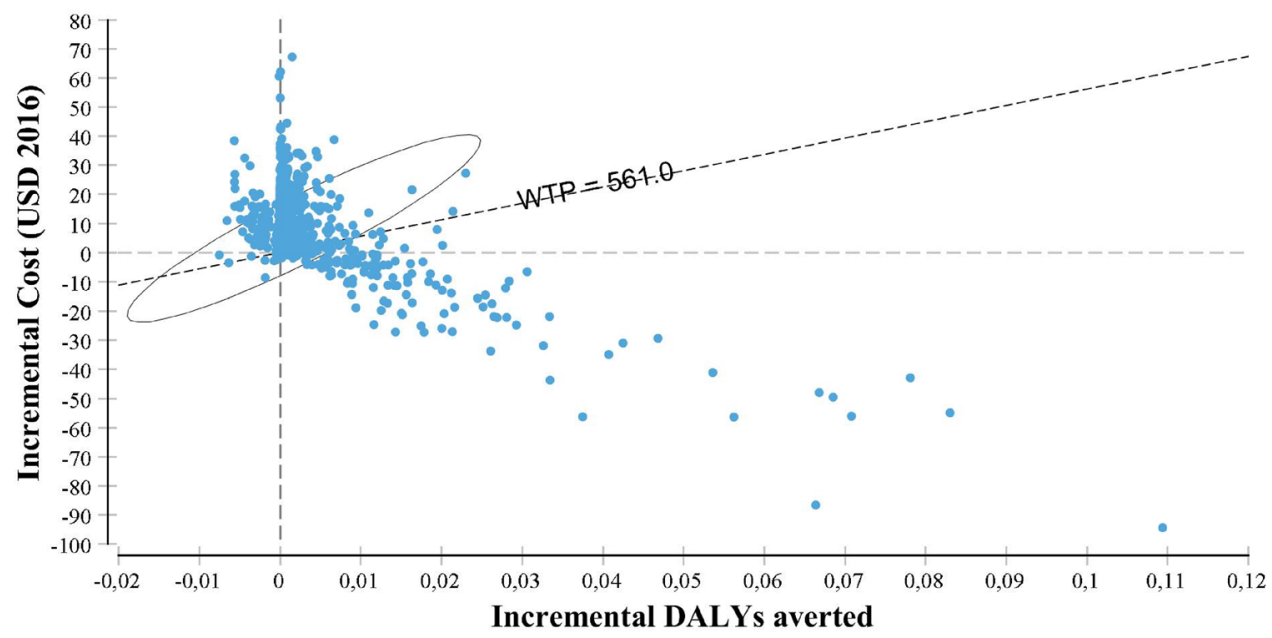

over microscopy. In scenario 2, LAMP becomes more competitive: about half of the simulations are located on or below the WTP threshold. The cost-effectiveness plane in scenario 3 suggests that RDT is significantly preferred over LAMP with nearly all simulations above the WTP threshold. Last, in scenario 4, most of the simulations are located above or on the WTP threshold: it appears more cost effective to first use RDT in the peripheral centers and perform microscopy on the negative patients at the reference clinic than directly performing LAMP (or microscopy) on all suspects at the reference clinic. However, as shown by the credible interval, the uncertainty regarding the cost effectiveness of this combined strategy is large.

With respect to the CEACs, as the effectiveness is close to 0 , different WTP values have little impact on modifying the probability of the tools to be cost effective and this further implies that for the tools to offer higher NMB, their respective costs must be reduced. In Scenario 1, where all tools are compared at the NMLCP level, RDT has a higher probability of being cost effective up to a WTP of a US\$1000 per DALY averted, which at first seems to contradict the finding in Table 3 that RDT is dominated. However, because the CEAC is based on the NMB of each option, it is possible for an option to have a higher net benefit without dominating another option [33]. The probability of RDT to be cost effective is even greater when employed at the peripheral level (i.e. scenario 3): regardless of the WTP value it has a $95 \%$ probability to be cost effective. In scenario 2 , when the LAMP capacity is maximised, the latter becomes more likely to be cost effective than RDT (at the reference clinic) above a WTP threshold of around US\$400 per DALY averted. In scenario 4, RDT performed at the peripheral level followed by microcopy is the strategy that is most likely to be cost effective irrespective of the WTP value (Table 4).

\section{Discussion}

This article discusses four hypothetical scenarios for microscopy, RDT and LAMP. That is, (1) the tools are implemented at the referral clinic (NMLCP), assuming the conservative approach that one test is performed at a time with LAMP; (2) the tools are used at the NMLCP but assuming the full capacity of the LAMP is reached (e.g. 48 tests processed at once); (3) microscopy and LAMP are implemented as in scenario 1 but RDT is implemented at peripheral levels, which translates into lower associated treatment costs to the patients; and (4) the tools are implemented as in scenario 3 , except that the CL suspects tested negative with RDT are sent to the reference clinic to be tested a second time with either microscopy or LAMP. In this last scenario, we assumed (as in scenario 3) that treatment is administered at the peripheral level. Such scenarios have been designed to capture the inherent benefits of the tools: the capacity of LAMP to process multiple samples at a time and the low level of expertise required for RDT, making its use possible in centers with no or low diagnostic capacities. However, its variable sensitivity may require a combined strategy with microscopy or LAMP, as illustrated in scenario 4.

In scenario 1, LAMP has a slightly higher effectiveness (i.e. lower associated DALYs) but the cost increase in using this tool compared with microscopy is around US\$6 per person tested. This higher cost implies lower NMB and would point to the use of microscopy as demonstrated by the Monte 
Fig. 6 Cost-effectiveness acceptability curves. The $y$-axis represents the probability of the tools being cost effective while the $x$-axis represents different willingness-to-pay values. LAMP Loopamp ${ }^{\mathrm{TM}}$ Leishmania Detection Kit, RDT CL Detect $^{\mathrm{TM}}$ Rapid Test

\section{Scenario 1}

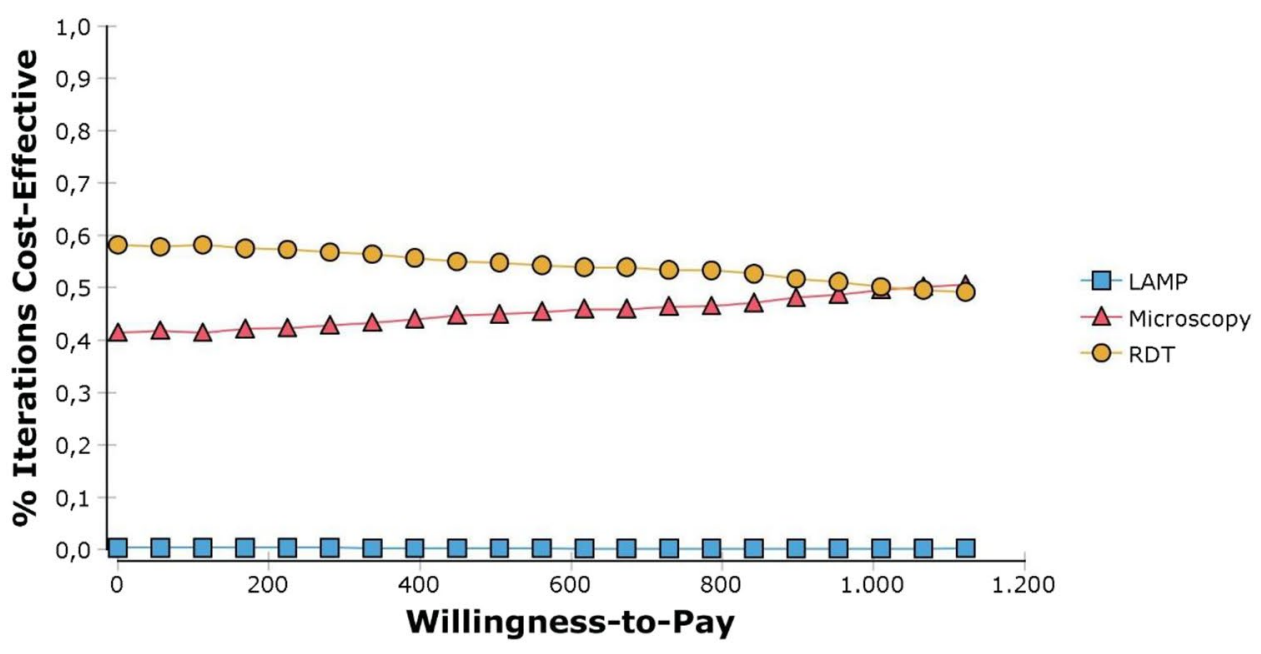

Scenario 2

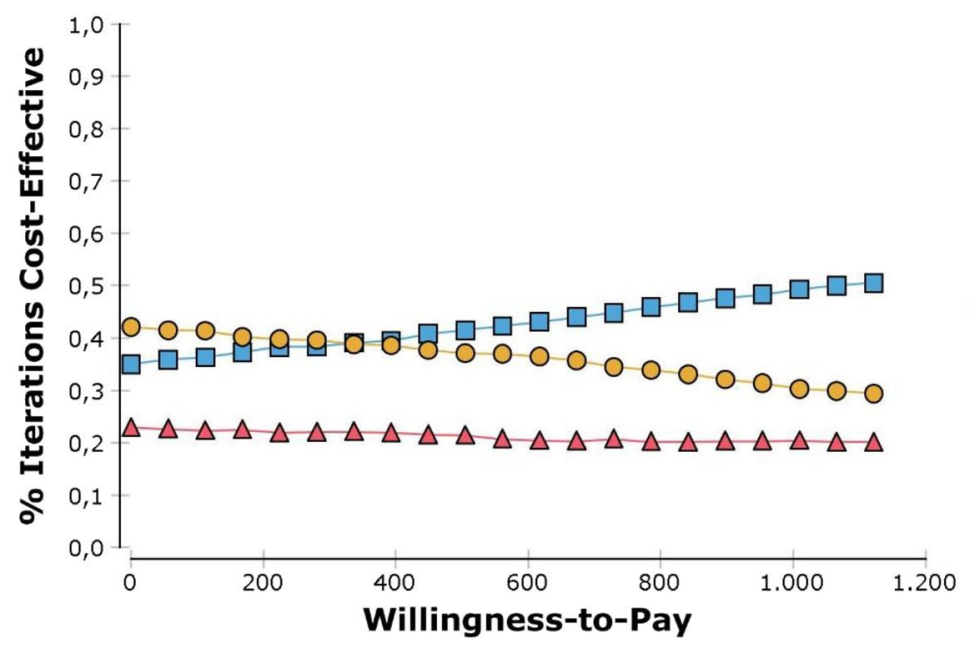

$\square$ - LAMP (full capacity) $\triangle$ Microscopy

- RDT

\section{Scenario 3}

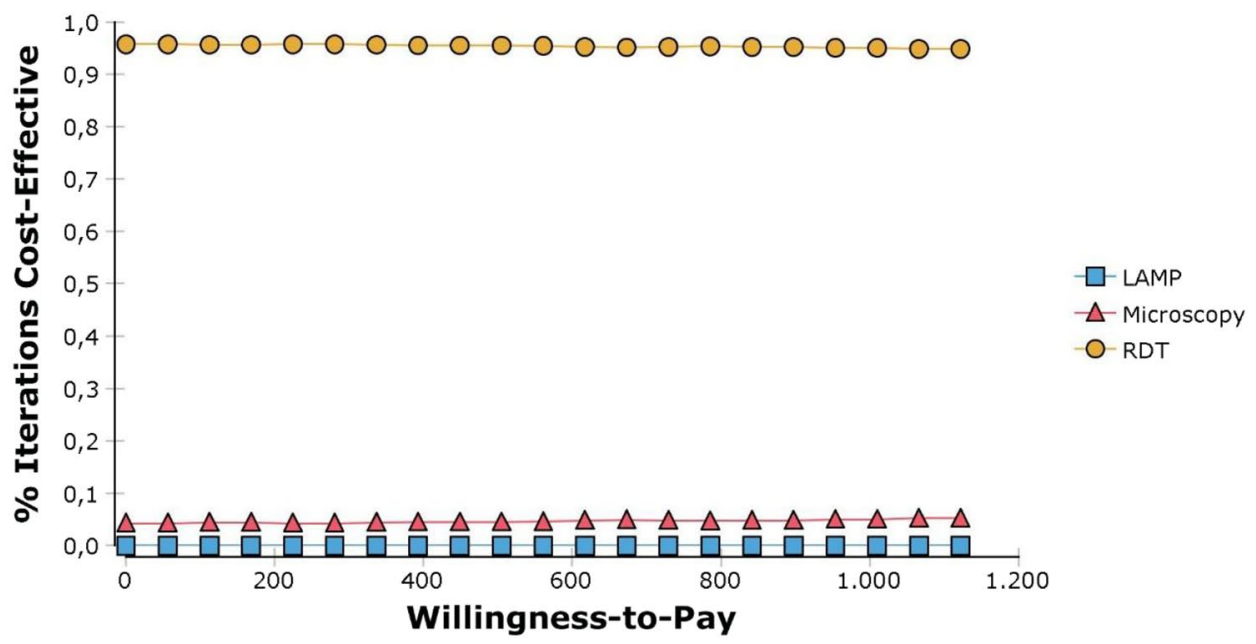


Fig. 6 (continued)

Scenario 4

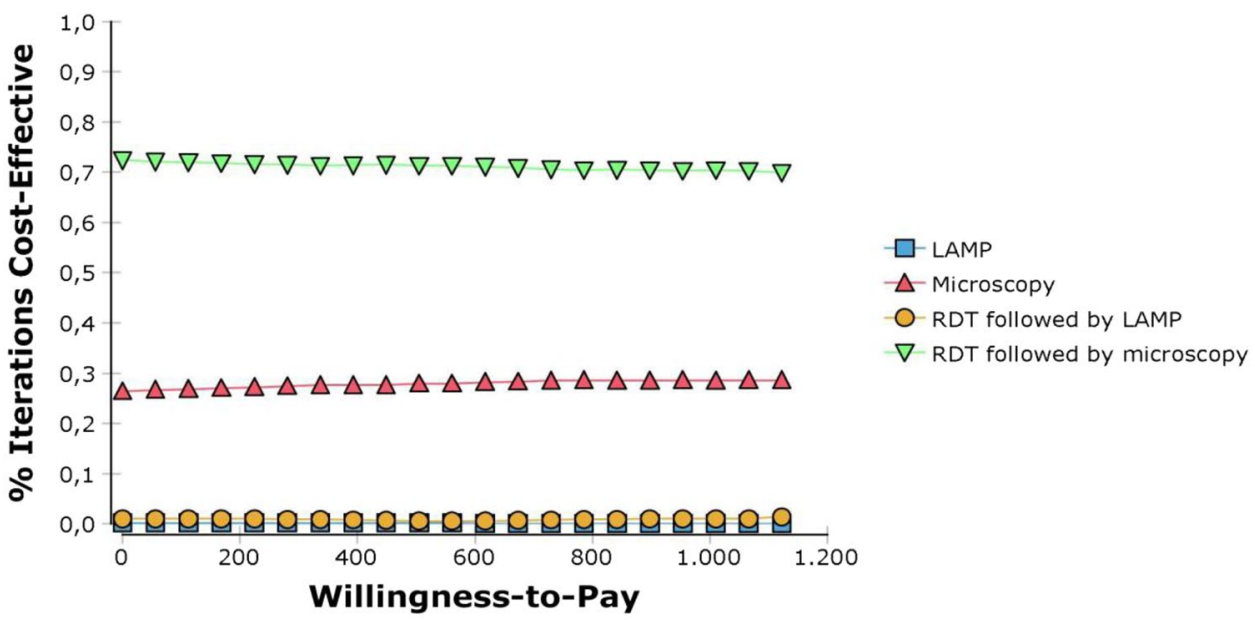

Carlo simulations. RDT has a very similar cost and effectiveness to microscopy, with crossing CEACs. In scenario 2, when dividing the labour cost on LAMP by 48 , its associated cost per person tested becomes slightly cheaper than that of RDT and microscopy. This scenario is plausible during the peak of the CL season or during an outbreak [34]. It also makes sense if LAMP is used simultaneously for other diseases than CL (e.g. malaria and/or tuberculosis). In such cases, LAMP potentially becomes a cost-effective solution but the uncertainty regarding the cost effectiveness of the latter vs. microscopy is significant, as demonstrated by the cost-effectiveness plane in Fig. 5.

In scenario 3, the associated treatment costs incurred by the patients, which exclusively include travel costs and wage losses associated with commuting and waiting times (as diagnosis and treatment is provided free of charge), were halved. The sensitivity analysis on this parameter shows that even if decreased by just 5\%, RDT becomes cheaper than any other strategy. We believe this is very likely to happen if the tool is implemented outside the reference clinic. However, one potential factor to consider if implementing the RDT in remote facilities is awareness-raising costs. As highlighted by the Ministry of Health, although the population may be aware that diagnostic and treatment options are provided in remote facilities, there is a general tendency to go to the reference clinic (NMLCP) even if it implies hours or days of travelling. Another feature to consider is the proportion of false negatives among CL individuals tested with RDT (i.e. sensitivity of $66.75 \%$ ).

This is considered in scenario 4, where RDT negative suspects (i.e. false positive and true negative) are tested at the reference clinic with LAMP or microscopy. RDT followed by LAMP is slightly more costly than RDT followed by microscopy and the difference in effectiveness between the two is negligible. This being said, it remains preferable to perform a primary screening of CL suspects remotely with RDT followed by microscopy or LAMP for the negative suspects, rather than testing all suspects directly with microscopy or LAMP (respectively) at the NMLCP. This is indeed illustrated by the CEACs in scenario 4: RDT followed by microscopy is on average $45-50 \%$ more likely to be cost effective than microscopy alone.

To conclude, the novel tools are promising and have their respective advantages. On the one hand, LAMP may be particularly relevant in a reference clinic during high endemic periods. Furthermore, in a context where laboratory expertise is lacking because of political instability and uncompetitive salaries, improving technology can significantly boost labour productivity. On the other hand, RDT may be particularly suitable in parts of the country where there is no/low diagnostic capacities. Such usage of RDT could be combined with additional testing at the reference clinic using other strategies with higher sensitivity. Nevertheless, even though this study attempts to capture the particularity of the tools with four different scenarios, one must keep in mind that estimates were collected from a single site, the NMLCP, which may not be representative of the whole country and which further implies that results may not be generalisable at the national level. Additionally, the results of this study are challenged by a very small incremental effectiveness across tools, questioning the use of DALYs when representing the burden associated with a disease such as CL. DALYs estimates based on the Global Burden of Diseases report may not capture the full burden, such as social stigmatisation and emotional burden that may be particularly important for women of young ages, and would suggest that, perhaps, the 


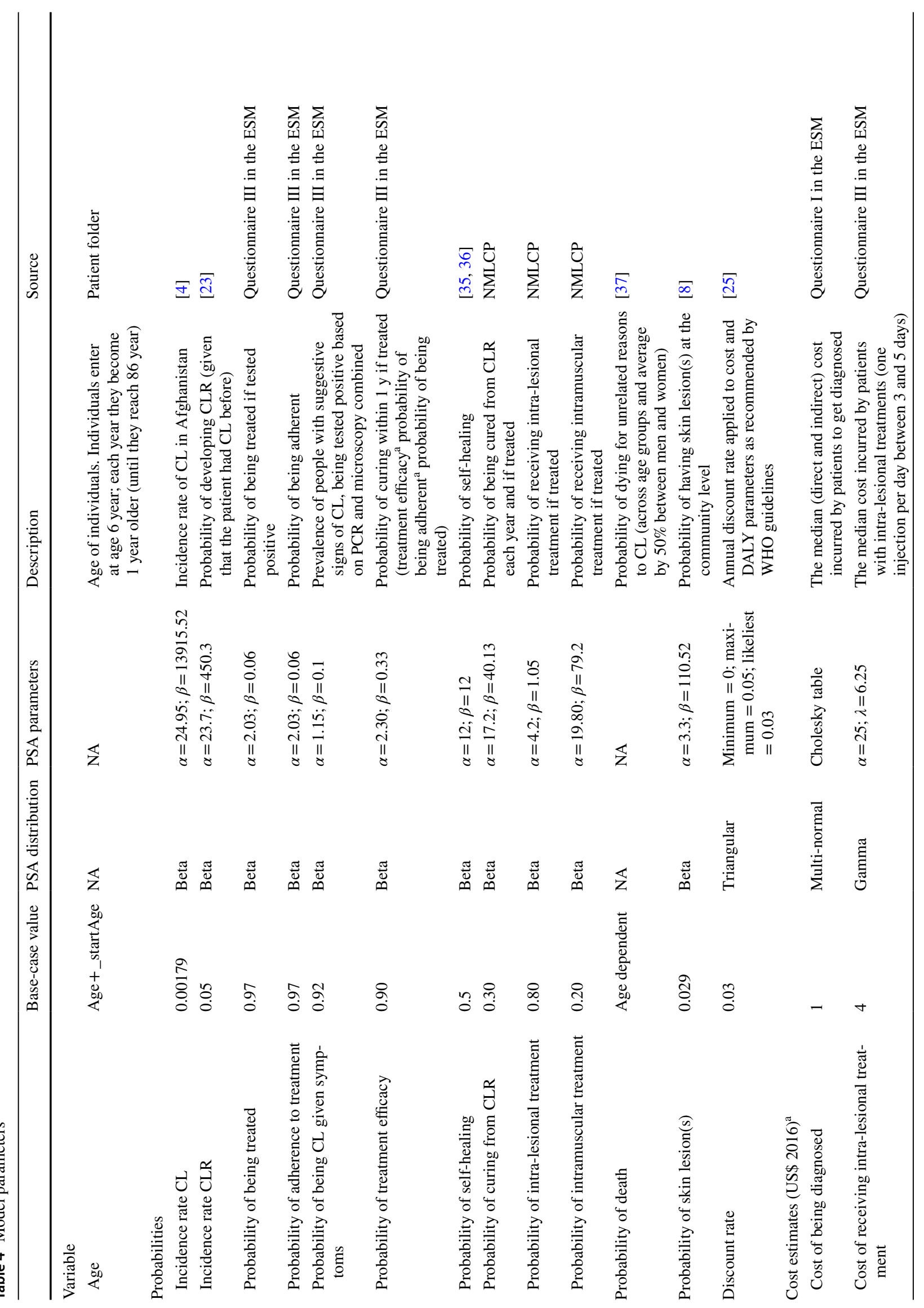




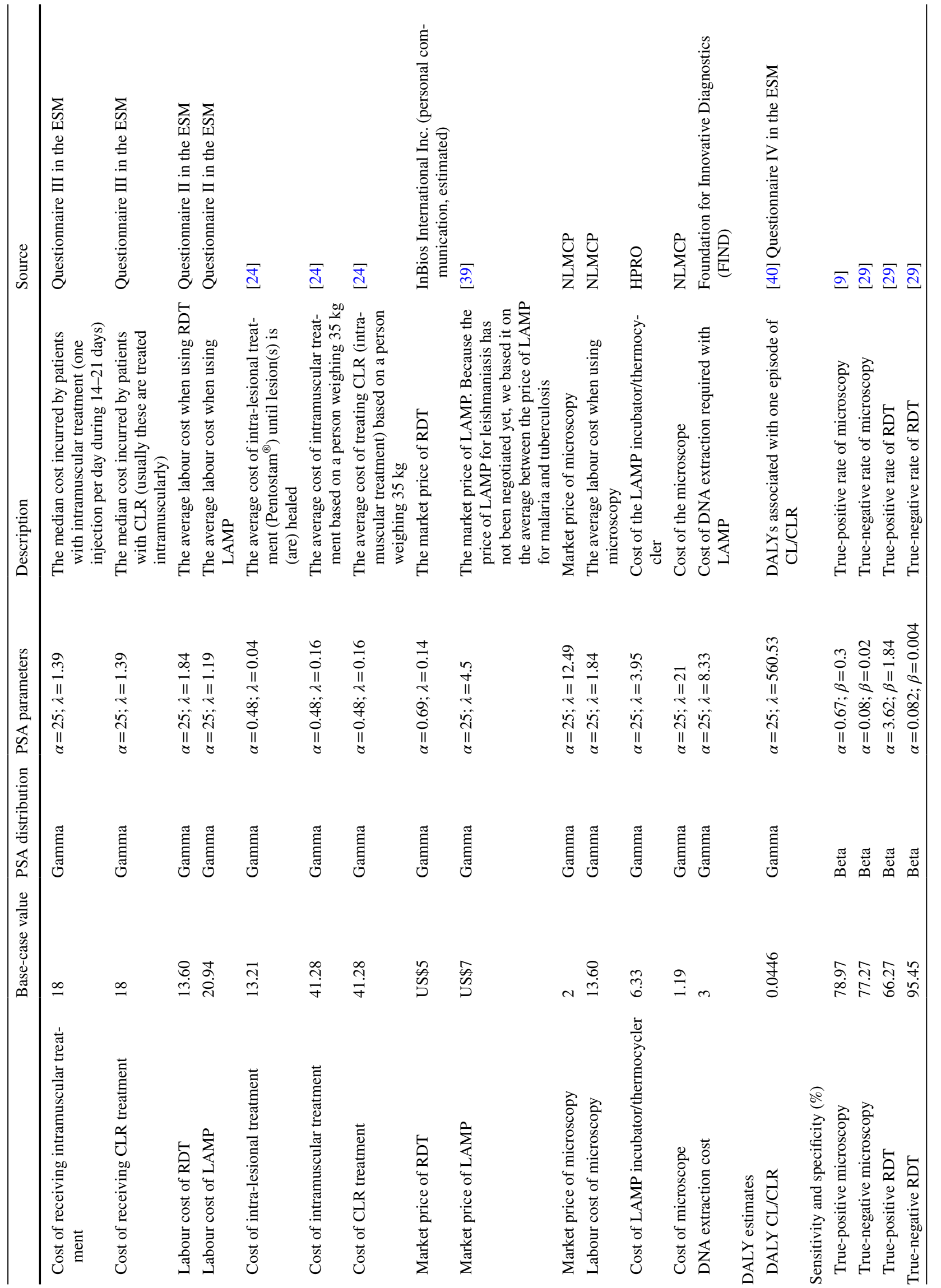


burden of CL should be measured in a more qualitative way, as attempted with the DLQI questionnaire.

\section{Compliance with Ethical Standards}

Funding This study was supported by funds from the European Union's Horizon 2020 Research and Innovation Program under the Marie Sklodowska-Curie Grant Agreement No. 642609, the Federal Ministry of Education and Research, Germany (KfW Grant Reference no. 202060457, Development of Products for the Prevention, Diagnosis and Treatment of Neglected and Poverty Related Diseases; https:// www.bmbf.de/en), the UK Aid from the UK Government, the Government of Switzerland and the Government of The Netherlands, which also contributed to the Foundation For Innovative New Diagnostics participation in this work.

Conflict of Interest Céline Aerts, Martijn Vink, Sayed Jalal Pashtoon, Sami Nahzat, Albert Picado, Israel Cruz and Elisa Sicuri have no conflicts of interest that are directly relevant to the contents of this article.

Ethics Approval The study was carried out in accordance with the Helsinki Declaration, and approved by the Institutional Review Board of the National Public Health Institute, Ministry of Public Health, Islamic Republic of Afghanistan (Approval No. 361549).

Consent to Participate The participants provided written informed consent in a one-on-one session with a member of the study. For illiterate individuals, the informed consent process was conducted in the presence of an impartial witness and for minors, the consent from a parent or guardian had to be obtained to be enrolled in the study.

Data Availability The datasets generated during and/or analysed during the current study are available in the figshare repository at https://doi. org/10.6084/m9.figshare.6949043.

Author Contributions IC, SP and SN provided the study concept and design, acquisition of data, analysis and interpretation, and study supervision. CA and ES contributed to the model design, data analysis and interpretation. CA, ES, IC, AP and MV contributed to the revision of the manuscript.

Open Access This article is distributed under the terms of the Creative Commons Attribution-NonCommercial 4.0 International License (http://creativecommons.org/licenses/by-nc/4.0/), which permits any noncommercial use, distribution, and reproduction in any medium, provided you give appropriate credit to the original author(s) and the source, provide a link to the Creative Commons license, and indicate if changes were made.

\section{References}

1. World Health Organization. Leishmaniasis: the disease. Geneva: World Health Organization;.

2. Kassi M, Kassi M, Afghan AK, Rehman R, Kasi PM. Marring leishmaniasis: the stigmatization and the impact of cutaneous leishmaniasis in Pakistan and Afghanistan. PLoS Negl Trop Dis. 2008;2(10):1-3. https://doi.org/10.1371/journal.pntd.0000259.

3. Bennis I, De Brouwere V, Belrhiti Z, Sahibi H, Boelaert M. Psychosocial burden of localised cutaneous leishmaniasis: a scoping review. BMC Public Health. 2018;18(1):1-12. https://doi. org/10.1186/s12889-018-5260-9. 
4. World Health Organization. Manual for case management of cutaneous leishmaniasis in the WHO Eastern Mediterranean Region. Geneva: World Health Organization; 2014.

5. World Health Organization. Weekly epidemiological record. Geneva: World Health Organization; 2016.

6. Berry I, Berrang-ford L. Leishmaniasis, conflict, and political terror: a spatio-temporal analysis. Soc Sci Med. 2016;167:140-9. https://doi.org/10.1016/j.socscimed.2016.04.038.

7. de Vries HJC, Reedijk SH, Schallig HDFH. Cutaneous leishmaniasis: recent developments in diagnosis and management. Am J Clin Dermatol. 2015;16(2):99-109. https://doi.org/10.1007/s4025 7-015-0114-z.

8. Boggild AK, Miranda-Verastegui C, Espinosa D, et al. Evaluation of a microculture method for isolation of Leishmania parasites from cutaneous lesions of patients in Peru. J Clin Microbiol. 2007;45(11):3680-4. https://doi.org/10.1128/JCM.01286-07.

9. Lineth A, Parrado R, De Doncker S, Dujardin J. American tegumentary leishmaniasis: direct species identification of Leishmania in non-invasive clinical samples. Trans R Soc Trop Med Hyg. 2007;101(4):368-71. https://doi.org/10.1016/j.trstm h.2006.06.009.

10. Reithinger R, Dujardin J-C, Louzir H, Pirmez C, Alexander $\mathrm{B}$, Brooker S. Cutaneous leishmaniasis. Lancet Infect Dis. 2007;7(9):581-96. https://doi.org/10.1016/S1473-3099(07)70209 -8 .

11. Masmoudi A, Hariz W, Marrekchi S, Amouri M, Turki H. Old World cutaneous leishmaniasis: diagnosis and treatment. J Dermatol Case Rep. 2013;7(2):31-41. https://doi.org/10.3315/ jdcr.2013.1135.

12. Oliveira LF, Schubach AO, Martins MM, et al. Systematic review of the adverse effects of cutaneous leishmaniasis treatment in the New World. Acta Trop. 2011;118(2):87-96. https:// doi.org/10.1016/j.actatropica.2011.02.007.

13. Notomi T, Mori Y, Tomita N, Kanda H. Loop-mediated isothermal amplification (LAMP): principle, features, and future prospects. J Microbiol. 2015;53(1):1-5. https://doi.org/10.1007/s1227 5-015-4656-9.

14. CL Detect ${ }^{\mathrm{TM}}$ Rapid Test for Cutaneous Leishmaniasis. InBios International Inc.; 2014

15. Refai WF, Madarasingha NP, Sumanasena B, et al. Efficacy, safety and cost-effectiveness of thermotherapy in the treatment of Leishmania donovani-induced cutaneous leishmaniasis: a randomized controlled clinical trial. Am J Trop Med Hyg. 2017;97(4):1120-6.

16. Chrusciak-talhari A, Dietze R, Talhari CC, et al. Randomized controlled clinical trial to access efficacy and safety of miltefosine in the treatment of cutaneous leishmaniasis caused by Leishmania (Viannia) guyanensis in Manaus. Brazil. Am J Trop Med Hyg. 2011;84(2):255-60. https://doi.org/10.4269/ajtmh.2011.10-0155.

17. Cardona-Arias JA, López-Carvajal L, Plata MPT, Vélez ID. Cost-effectiveness analysis of thermotherapy versus pentavalent antimonials for the treatment of cutaneous leishmaniasis. J Evid Based Med. 2017;10(2):81-90.

18. Reithinger R, Coleman PG. Treating cutaneou leishmaniasis patients in Kabul, Afghanistan: cost-effectiveness of an operational program in a complex emergency setting. BMC Infect Dis. 2007;7:1-9. https://doi.org/10.1186/1471-2334-7-3.

19. Stahl H-C, Ahmadi F, Nahzat SM, Dong H-J, Stahl K-W, Sauerborn R. Health economic evaluation of moist wound care in chronic cutaneous leishmaniasis ulcers in Afghanistan. Infect Dis Poverty. 2018;7(1):12. https://doi.org/10.1186/s4024 9-018-0389-4.

20. Boggild AK, Valencia BM, Espinosa D, et al. Detection and species identification of leishmania DNA from filter paper lesion impressions for patients with American cutaneous leishmaniasis. Clin Infect Dis. 2010;50(1):e1-6. https://doi.org/10.1086/64873 0 .
21. Bensoussan E, Nasereddin A, Jonas F, Schnur LF, Jaffe CL. Comparison of PCR assays for diagnosis of cutaneous leishmaniasis. J Clin Microbiol. 2006;44(4):1435-9. https://doi.org/10.1128/ JCM.44.4.1435.

22. Barrio A, Mora MC, Ramos F, Moreno S, Samson R, Basombrío MA. Short report: use of kDNA-based polymerase chain reaction as a sensitive and differentially diagnostic method of American tegumentary Leishmaniasis in disease-endemic areas of northern Argentina. Am J Trop Med Hyg. 2007;77(4):636-9.

23. June P, Modabber F, Buffet PA, Torreele E, Milon G, Croft SL. Consultative meeting to develop a strategy for treatment of cutaneous leishmaniasis. Institute Pasteur; 13-15, Paris. Kinetoplastid biology and disease. 2006;24(2007):1-24. https://doi. org/10.1186/1475-9292-6-3.

24. World Health Organization. Annex 6. Costs of medicines in current use for the treatment of leishmaniasis. Table A6.1: drug prices (January 2010). Geneva: World Health Organization; 2010.

25. World Health Organization. WHO guide to cost-effectiveness analysis. Geneva: World Health Organization; 2003.

26. Finlay AY, Khan GK. Dermatology Life Quality Index (DLQI): a simple practical measure for routine clinical use. Clin Exp Dermatol. 1994;19(3):210-6. https://doi.org/10.1111/j.1365-2230.1994. tb01167.x.

27. Global Burden of Disease Study 2015. Disability weights. Seattle (WA):; 2016.

28. World Health Organization. WHO methods and data sources for global burden of disease estimates 2000-2015. Geneva: World Health Organization; 2017.

29. Vink MMT, Nahzat SM, Rahimi H, et al. Evaluation of point-ofcare tests for cutaneous leishmaniasis diagnosis in Afghanistan. EBioMedicine. 2018 Nov 2. pii: S2352-3964(18)30483-3. https ://doi.org/10.1016/j.ebiom.2018.10.063. [Epub ahead of print].

30. Bertram MY, Lauer JA, Joncheere K De, et al. WHO: Cost-effectiveness thresholds: pros and cons. 2016.

31. Briggs AH. Statistical approaches to handling uncertainty in health economic evaluation. Eur J Gastroenterol Hepatol. 2004;16(6):55161. https://doi.org/10.1097/01.meg.0000108347.41221.50.

32. Nayagam S, Conteh L, Sicuri E, et al. Cost-effectiveness of community-based screening and treatment for chronic hepatitis B in The Gambia: an economic modelling analysis. Lancet Glob Health. 2016;4(8):e568-78. https://doi.org/10.1016/S2214 $-109 X(16) 30101-2$.

33. Barton GR, Briggs AH, Fenwick EAL. Optimal cost-effectiveness decisions: the role of the cost-effectiveness acceptability curve (CEAC), the cost-effectiveness acceptability frontier (CEAF), and the expected value of perfection information (EVPI). Value Health. 2008;11(5):886-97. https://doi.org/10.11 11/j.1524-4733.2008.00358.x.

34. Faulde MK, Heyl G, Mohammed A. Zoonotic cutaneous. The Public Health Image Library. Emerg Infect Dis. 2006;12(10)

35. World Health Organization. Control of the leishmaniases: report of a meeting of the WHO Expert Committee on the control of leishmaniases. Geneva: World Health Organization; 2010.

36. Hepburn NC. Cutaneous leishmanaisis: an overview. J Postgrad Med. 2003;49(1):50-4.

37. Afghan Public Health Insitute, Central Statistics Organization, Indian Institute of Health Management Research, World Health Organization. Afghanistan Mortality Survey 2010. 2011.

38. Reithinger R, Mohsen M, Aadil K, Sidiqi M, Erasmus P, Coleman PG. Anthroponotic cutaneous leishmaniasis, Kabul. Afghanistan. Emerg Infect Dis. 2003;9(6):727-9. https://doi.org/10.3201/eid09 06.030026.

39. Foundation For Innovative New Diagnostics (FIND). Loopamp ${ }^{\mathrm{TM}}$ (LAMP) for TB and malaria.

40. Global Burden of Disease Study 2015. Disability weights. Seattle (WA); 2016 